\title{
Interim estimates of influenza vaccine effectiveness in 2012/13 from Canada's sentinel surveillance network, January 2013
}

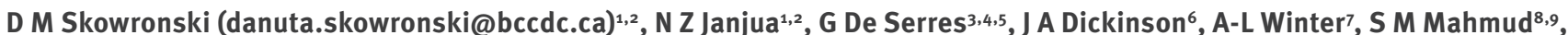

S Sabaiduc ${ }^{1}$, J B Gubbay ${ }^{7,10}$, H Charest ${ }^{3}$, M Petric ${ }^{1,2}$, K Fonseca ${ }^{6,11}$, P Van Caeseele ${ }^{12}$, T L Kwindt ${ }^{1,2}$, M Krajden ${ }^{1,2}$, A Eshaghi7 , Y Li ${ }^{9,13}$

1. British Columbia Centre for Disease Control, Vancouver, Canada

2. University of British Columbia, Vancouver, Canada

3. Institut National de Santé Publique du Québec (National Institute of Health of Quebec), Québec, Canada

4. Laval University, Quebec, Canada

5. Centre Hospitalier Universitaire de Québec (University Hospital Centre of Quebec), Québec, Canada

6. University of Calgary, Calgary, Canada

7. Public Health Ontario, Toronto, Canada

8. Winnipeg Regional Health Authority, Winnipeg, Canada

9. University of Manitoba, Winnipeg, Canada

10. University of Toronto, Toronto, Canada

11. Alberta Provincial Laboratory, Calgary, Canada

12. Cadham Provincial Laboratory, Winnipeg, Canada

13. National Microbiology Laboratory, Public Health Agency of Canada, Winnipeg, Canada

Citation style for this article:

Skowronski DM, Janjua NZ, De Serres G, Dickinson JA, Winter A, Mahmud SM, Sabaiduc S, Gubbay JB, Charest H, Petric M, Fonseca K, Van Caeseele P, Kwindt TL, Krajden M, Eshaghi A, Li Y. Interim estimates of influenza vaccine effectiveness in 2012/13 from Canada's sentinel surveillance network, January 2013 . Euro Surveill. 2013;18(5):pii=20394. Available online: http://www.eurosurveillance.org/ViewArticle.aspx?Articleld=20394

The 2012/13 influenza season in Canada has been characterised to date by early and moderately severe activity, dominated (90\%) by the $\mathrm{A}\left(\mathrm{H}_{3} \mathrm{~N}_{2}\right)$ subtype. Vaccine effectiveness (VE) was assessed in January 2013 by Canada's sentinel surveillance network using a test-negative case-control design. Interim adjustedVE against medically attended laboratory-confirmed influenza $\mathrm{A}\left(\mathrm{H}_{3} \mathrm{~N}_{2}\right)$ infection was $45 \%$ (95\% $\mathrm{Cl}$ : 13-66). Influenza $\mathrm{A}\left(\mathrm{H}_{3} \mathrm{~N}_{2}\right)$ viruses in Canada are similar to the vaccine, based on haemagglutination inhibition; however, antigenic site mutations are described in the haemagglutinin gene.

\section{Background}

The 2012/13 influenza season in North America has shown moderately severe activity, spiking over the December/January holiday period, with influenza $\mathrm{A}\left(\mathrm{H}_{3} \mathrm{~N}_{2}\right)$ viruses predominating among typed/subtyped viruses to date in both Canada (about 90\%) and the United States (US) (about 70\%) [1,2].

The updated 2012/13 $\mathrm{A}\left(\mathrm{H}_{3} \mathrm{~N}_{2}\right)$ reference strain recommended by the World Health Organization as vaccine component for the northern hemisphere (A/ Victoria/361/2011-like) is antigenically distinct from that recommended for the previous season (A/ Perth/16/2009-like) [3], with 11 amino acid (AA) residue differences at antigenic sites of the haemagglutinin (HA) surface protein [4].

Vaccine effectiveness (VE) in Canada was assessed by the country's sentinel surveillance network in January
2013. Here we report the interim 2012/13 VE estimates against the dominant circulating influenza $A\left(\mathrm{H}_{3} \mathrm{~N}_{2}\right)$ subtype in the context of antigenic and genetic characterisation of circulating strains.

\section{Estimating influenza vaccine effectiveness}

As previously described [5-11], a test-negative casecontrol design was used to estimate VE, whereby a patient presenting with influenza-like illness (ILI) testing positive for influenza virus was considered a case and a person testing negative was considered a control.

Several hundred community-based practitioners in sentinel surveillance sites across participating provinces (British Columbia, Alberta, Manitoba, Ontario and Quebec) may offer nasal or nasopharyngeal swabbing to any patient presenting within seven days of symptom onset of ILI - defined as acute onset of respiratory illness with fever and cough and one or more of the following: sore throat, arthralgia, myalgia or prostration.

The VE analysis period included specimens collected from 1 November 2012 (week 44: 28 October 2012-3 November 2012) to 23 January 2013 (week 4: 20-26 January 2013), taking into account onset of influenza activity (Figure 1) and an immunisation campaign that started in October. Epidemiological information was obtained from consenting patients or their parents/ guardians using a standard questionnaire at the time of specimen collection, before testing. Ethics review boards in each participating province approved this study. 
Specimens were tested for influenza viruses A (to subtype level) and $B$ at provincial reference laboratories by real-time reverse-transcription polymerase chain reaction according to provincial protocols $[4,11]$. Odds ratios (OR) for influenza vaccination among cases versus controls were estimated by multivariable logistic regression. VE against medically attended laboratory-confirmed influenza was calculated as [1 - OR] $\times$ 100. Patients for whom the timing of vaccination was unknown or was less than two weeks before symptom onset were excluded from the primary VE analysis but explored in sensitivity analyses. Those with unknown comorbidity were included and further explored in sensitivity analyses.

\section{Genetic characterisation of sentinel influenza $A(H 3 N 2)$ viruses}

Sequencing of the $\mathrm{HA}_{1}$ gene of a convenience sample $(n=82)$ of available influenza $A\left(\mathrm{H}_{3} \mathrm{~N}_{2}\right)$ viruses, spanning the season so far but with emphasis on more recent activity, was undertaken for each province to identify AA substitutions within the 131 residues of antigenic sites $A-E[11,12]$. These were expressed as percentage identity and relatedness compared with the vaccine reference strain (A/Victoria/351/2011). Pairwise identities were calculated from alignments of translated protein sequences generated in Geneious Pro v4.8.5 using a MUSCLE multiple sequence alignment algorithm. The approximate likelihood method was used to generate the phylogenetic tree of aligned nucleotide sequences in Geneious Pro v4.8.5.

HA sequences from reference strains used in the phylogenetic analysis were obtained from the EpiFlu database of the Global Initiative on Sharing Avian Influenza Data (GISAID) (Table 1).

\section{Interim estimates of influenza vaccine effectiveness}

\section{Participants}

A total of 939 specimens were submitted from sentinel surveillance sites between 1 November 2012 and 23 January 2013. After exclusion criteria were applied (Figure 2), 739 participants contributed to overall VE analysis: their profile was similar to that seen in VE

\section{FIGURE 1}

Laboratory detection of influenza by week and virus subtype, Canada, 2012/13 sentinel surveillance system ( $\mathrm{n}=833$ )

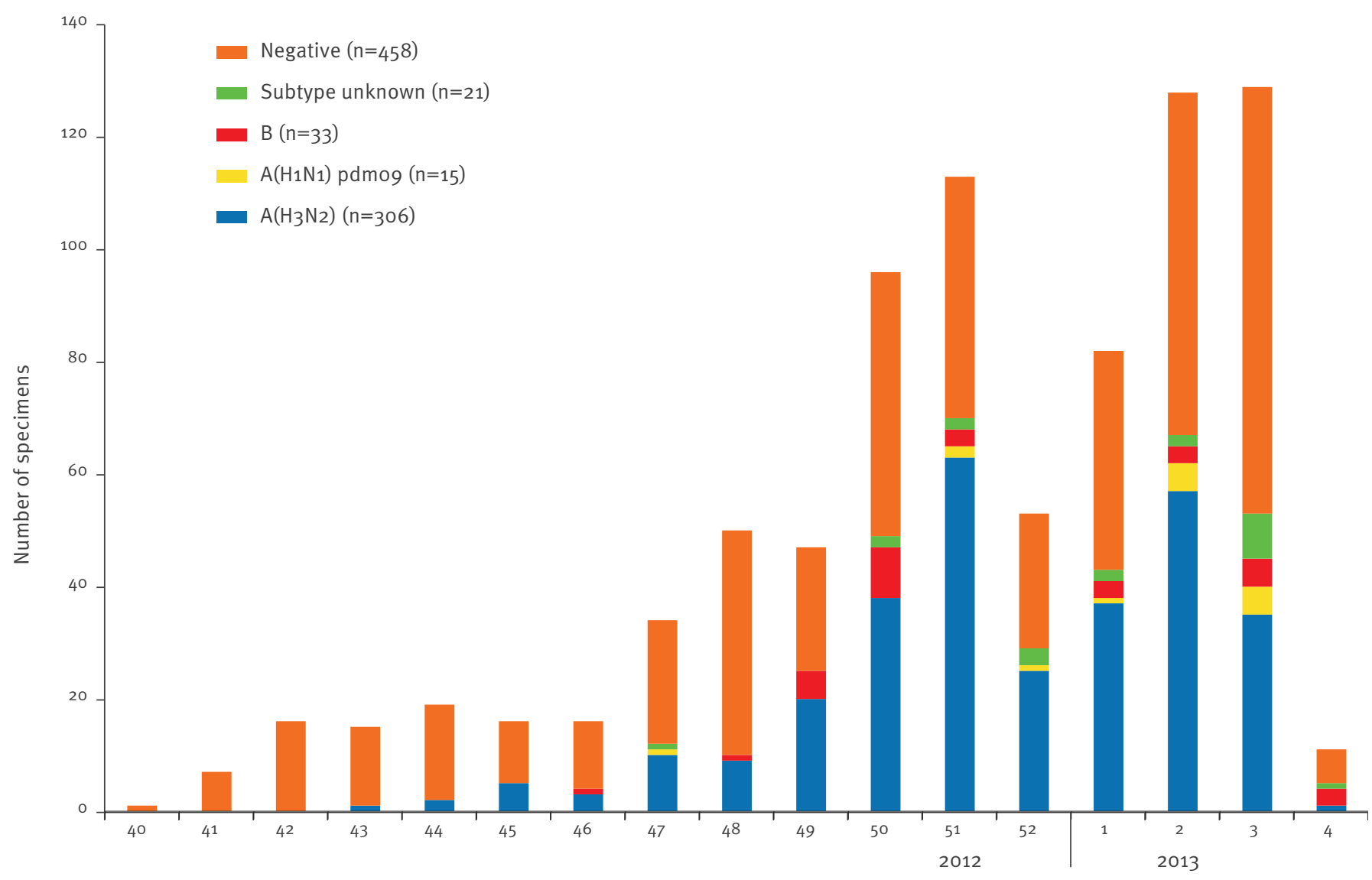

Week number

Of 999 nasal or nasopharyngeal specimens collected between 1 October 2012 (week 40: 30 September-6 October 2012) and 23 January 2013 (week 4: 20-26 January 2013), we excluded from the epidemic curve specimens from the following patients: those failing to meet the influenza-like illness (ILI) case definition or for whom it was unknown $(n=24)$; those whose specimens were collected more than seven days after symptom onset or for whom the interval was unknown $(n=132)$; those whose age was unknown ( $n=1)$ and those for whom influenza test results were unavailable or indeterminate $(n=9)$. Specimens were included regardless of the patient's vaccination status or timing of vaccination; specimens from patients with unknown comorbidity were also included. 


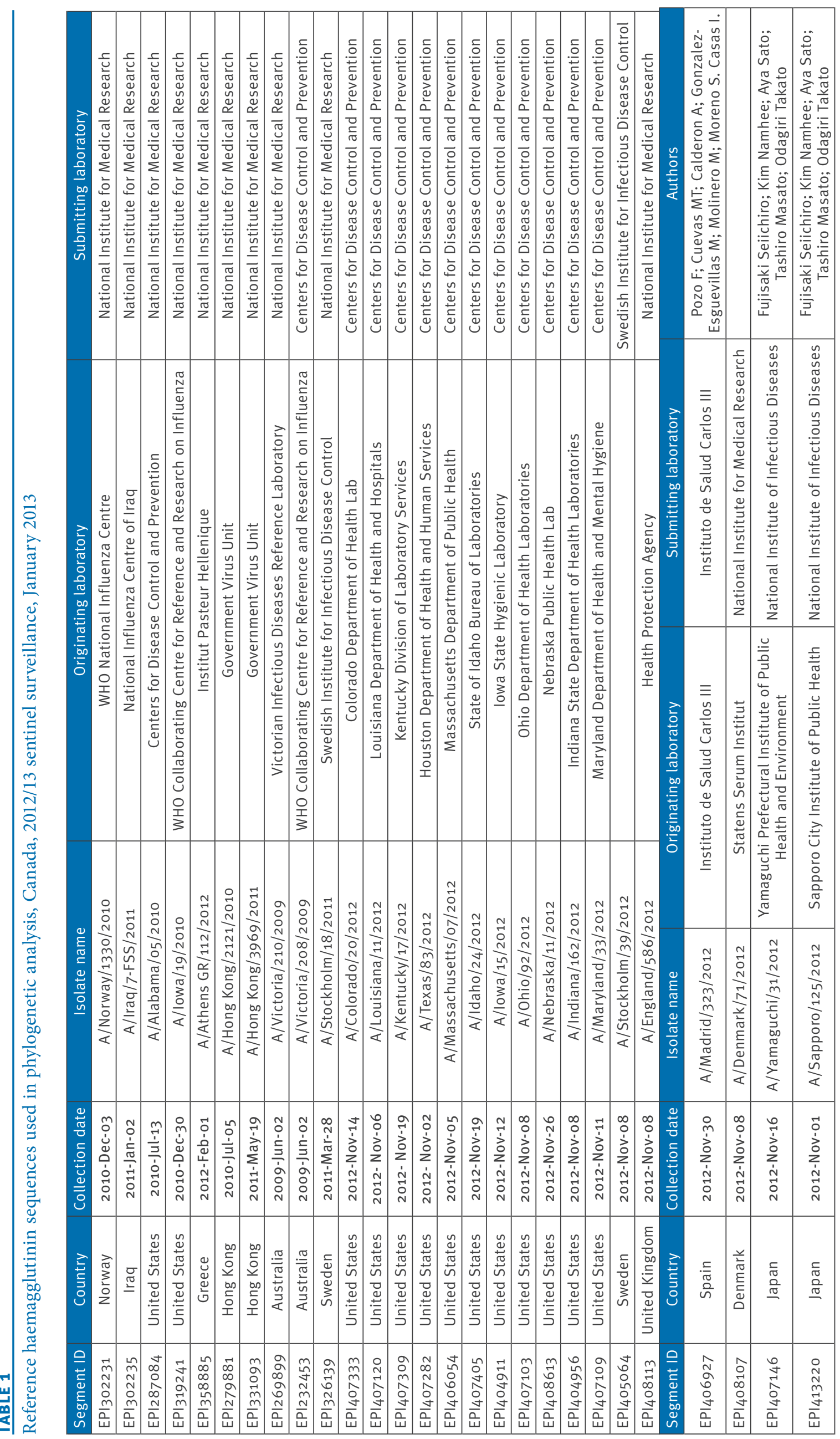


analyses of previous seasons $[4,8,9,11]$. Those aged 20-49 years contributed most to the analysis (43\%) and the median interval between symptom onset and specimen collection was three days (Table 2 ).

About half $(355 / 739)$ of the specimens were positive for influenza, of which $86 \%$ (287/334) of subtyped viruses were $\mathrm{A}\left(\mathrm{H}_{3} \mathrm{~N}_{2}\right)$ (Table 3 ), a predominance similar to that noted elsewhere for Canada (Figure 1) [1]. The $2012 / 13$ vaccine was received by $27 \%$ (108/402) controls (i.e. test-negative) and $17 \%(61 / 365)$ cases (i.e. test-positive) (p<0.001) (Table 2). Of those with information available for both 2011/12 and 2012/13 $(n=682), 136 / 150$ (91\%) of those immunised in 2012/13 were also immunised in 2011/12. The proportion of controls reporting immunisation for 2012/13 and earlier seasons was comparable to that in previous sentinel and other survey reports for Canada (about 30\%) $[4,7-9,11,14]$ and was also similar for influenza $A\left(\mathrm{H}_{1} \mathrm{~N}_{1}\right)$ pdmo9 immunisation: $48 \%$ compared with previous Canadian surveys (41\%) [11]. The proportion of samples from patients with comorbidity was comparable to previous sentinel system estimates (14-23\%) and other reports for Canada (15-20\%) [4,7-11,15].

The overall crude (unadjusted) VE against influenza $\mathrm{A}\left(\mathrm{H}_{3} \mathrm{~N}_{2}\right)$ virus was $39 \%(95 \% \mathrm{Cl}: 10-59)$ and against any influenza was $45 \%$ (95\% Cl: 20-63) (Table 4). Fully adjusted VEs were $45 \%(95 \% \mathrm{Cl}: 13-66)$ for $\mathrm{A}\left(\mathrm{H}_{3} \mathrm{~N}_{2}\right)$ and $52 \%(95 \% \mathrm{Cl}: 25-69)$ overall. The overall VE estimate reflects the predominance of influenza $A\left(\mathrm{H}_{3} \mathrm{~N}_{2}\right)$

\section{FIGURE 2}

Specimen exclusion for interim influenza vaccine effectiveness analysis, Canada, 2012/13 sentinel surveillance system

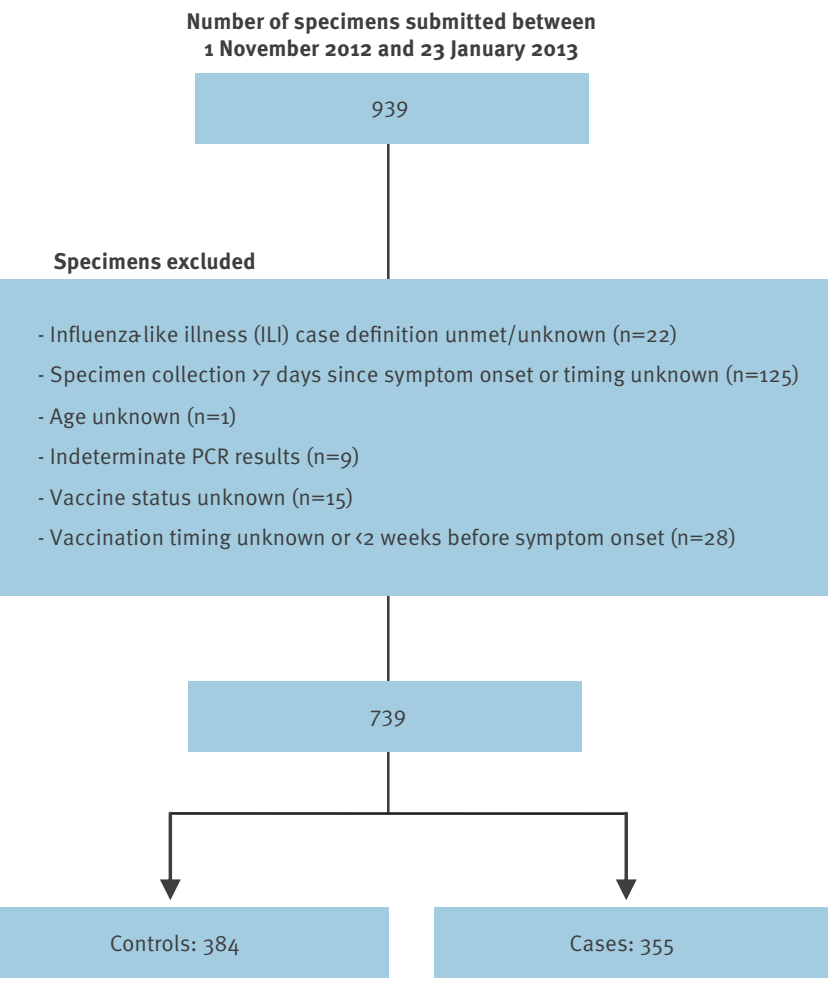

virus, with little contribution from influenza $B$ or $A\left(\mathrm{H}_{1} \mathrm{~N}_{1}\right)$ viruses, precluding reliable estimates for those components.

\section{Virus characterisation}

All influenza $A\left(\mathrm{H}_{3} \mathrm{~N}_{2}\right)$ isolates to date this season characterised in Canada by the haemagglutination inhibition assay have been considered antigenically similar to the $2012 / 13$ vaccine component, although characterisation so far includes few $(n=3)$ of the sentinel viruses described here [1]. HA1 sequences of a subset of 82 (29\%) sentinel $\mathrm{A}\left(\mathrm{H}_{3} \mathrm{~N}_{2}\right)$ viruses were thus assessed for substitutions potentially contributing to suboptimal VE (Figure 3, Table 5). Sequencing was based on original specimens from British Columbia $(n=15)$, Alberta $(n=25)$, Manitoba $(n=4)$ and Ontario $(n=11)$ and virus isolates from Quebec $(n=27)$.

Of the 82 sequences, 75 clustered within the European Centre for Disease Prevention and Control (ECDC)-described Clade $3 C$, which includes the $A$ / Victoria/361/2011 vaccine strain (Figure 3) [16]. There were, however, four to eight AA mutations (93.9-96.9\% vaccine identity) in $\mathrm{HA}_{1}$ antigenic sites compared with the $A / V i c t o r i a / 361 / 2011$ vaccine reference strain as follows: 2/82 with four AA mutations (from specimens collected mid-November and mid-December); 19/82 with five (October-January); 22/82 with six (OctoberJanuary); 29/82 with seven (November-January) and $3 / 82$ with eight mutations (late-December). Of note, the $32 / 82$ viruses with seven or eight $A A$ mutations included loss of glycosylation through T128A substitution in antigenic site $B$. The remaining seven sentinel sequences (collected mid-November to early January) clustered within ECDC Clade 6 (A/lowa/19/2010-like) with $6 / 82$ showing $11 \mathrm{AA}$ mutations ( $91.6 \%$ vaccine identity) and one exhibiting 12 AA mutations (90.8\% vaccine identity) relative to the $A /$ Victoria/361/2011 vaccine strain (Figure 3, Table 5). These Clade 6 viruses also included loss of glycosylation at position $\mathrm{N}_{45} \mathrm{~S}$, a non-antigenic site mutation.

\section{Discussion}

Mid-season reporting of virus evolution, vaccine relatedness and VE can support real-time risk communication and mitigation. Our interim 2012/13 VE results show that vaccination reduced the risk of medically attended laboratory-confirmed influenza due to the predominant $\mathrm{A}\left(\mathrm{H}_{3} \mathrm{~N}_{2}\right)$ virus subtype by about half.

Our estimates are comparable to, if somewhat lower than, interim 2012/13 VE estimates recently reported by the US indicating $62 \%$ VE overall, $55 \%$ for influenza $A$ and $70 \%$ for influenza $B$ [17]. The proportion of influenza A viruses contributing to interim VE analysis in the US study setting ( $57 \%$ ) is different from the profile for the rest of the US (about 70\%) or Canada (about $90 \%)$; influenza $\mathrm{A}\left(\mathrm{H}_{3} \mathrm{~N}_{2}\right)$ viruses have so far predominated in both countries $[1,2]$. Participant profiles were not presented and multivariable adjustment was also not undertaken in the interim US analysis. Although 


\section{TABLE 2}

Profile of participants included in primary analysis, interim 2012/13 influenza vaccine effectiveness evaluation, Canada

\begin{tabular}{|c|c|c|c|}
\hline Characteristics & $\begin{array}{c}\text { Control (test-negative) } \\
\mathrm{N}=384 \\
\mathrm{n}(\%)\end{array}$ & $\begin{array}{c}\text { Case (test-positive) } \\
\mathrm{N}=355 \\
\mathrm{n}(\%)\end{array}$ & $\begin{array}{l}\text { Total } \\
\mathrm{N}=739 \\
\mathrm{n}(\%)\end{array}$ \\
\hline \multicolumn{4}{|l|}{ Age group (years) } \\
\hline $1-8$ & $59(15)$ & 67 (19) & $126(17)$ \\
\hline 9-19 & $38(10)$ & $46(13)$ & $84(11)$ \\
\hline $20-49$ & $166(43)$ & $149(42)$ & $315(43)$ \\
\hline $50-64$ & $80(21)$ & $61(17)$ & $141(19)$ \\
\hline$\geq 65$ & $41(11)$ & $32(9)$ & $73(10)$ \\
\hline Median (range) & $37((1-92)$ & $32((1-90)$ & $35((1-92)$ \\
\hline \multicolumn{4}{|l|}{ Sex } \\
\hline Female & $228(59)$ & $206(58)$ & $434(59)$ \\
\hline \multicolumn{4}{|l|}{ Comorbidity $^{a}$} \\
\hline No & $270(70)$ & $271(76)$ & $541(73)$ \\
\hline Yes & $81(21)$ & $61(17)$ & $142(19)$ \\
\hline Unknown & $33(9)$ & $23(6)$ & $56(8)$ \\
\hline \multicolumn{4}{|l|}{ Received 2012/13 TIVb,c } \\
\hline Any immunisation ${ }^{d}$ & $108 / 402(27)$ & $61 / 365(17)$ & $169 / 767(22)$ \\
\hline$\geq 2$ weeks before symptom onset & $90(23)$ & $51(14)$ & $141(19)$ \\
\hline Among those with comorbidity & $28(35)$ & $20(33)$ & $48(34)$ \\
\hline Among those without comorbidity & $55(20)$ & $29(11)$ & $84(16)$ \\
\hline \multicolumn{4}{|l|}{ Received 2011/12 TIVe } \\
\hline No & $227(69)$ & $240(73)$ & $467(71)$ \\
\hline Yes & $104(31)$ & $88(27)$ & $192(29)$ \\
\hline \multicolumn{4}{|l|}{ Received 2010/11 TIV } \\
\hline No & $204(64)$ & $217(70)$ & $421(67)$ \\
\hline Yes & $113(36)$ & $91(30)$ & $204(33)$ \\
\hline \multicolumn{4}{|c|}{ Received adjuvanted $\mathrm{A}\left(\mathrm{H}_{1} \mathrm{~N}_{1}\right)$ pdmog vaccine $\mathrm{v}^{\mathrm{g}}$} \\
\hline No & $156(52)$ & $147(51)$ & $303(52)$ \\
\hline Yes & $144(48)$ & $140(49)$ & $284(48)$ \\
\hline \multicolumn{4}{|l|}{ Specimen collection interval (days) } \\
\hline$\leq 4$ & $282(73)$ & $293(83)$ & $575(78)$ \\
\hline $5-7$ & $102(27)$ & $62(17)$ & $164(22)$ \\
\hline Median (range) & $3(0-7)$ & $3(0-7)$ & $3(0-7)$ \\
\hline
\end{tabular}

TIV: trivalent influenza vaccine.

a Chronic medical conditions that place individuals at higher risk of serious complications (hospitalisation or death) from influenza as defined by Canada's National Advisory Committee on Immunization [13], including heart, pulmonary (including asthma), renal, metabolic (such as diabetes), blood, cancer, immune compromising conditions or those that compromise the management of respiratory secretions and increase the risk of aspiration or morbid obesity. Questionnaire was answered as 'yes', 'no' or 'unknown' to any one or more of these conditions without specifying.

b Vaccine status was based on self/parental/guardian report. Detail related to special paediatric dosing requirements was not sought. Immunised participants were predominantly offered split (non-adjuvanted) 2012/13 trivalent inactivated influenza vaccine during the regular autumn immunisation campaign. In British Columbia and Quebec, influenza vaccine is provided free of charge to high-risk groups [13]. Others are encouraged to receive vaccine but must purchase it. In Ontario, Alberta and Manitoba, the vaccine is provided free of charge to all citizens aged $\geq 6$ months.

In Canada, adjuvanted vaccine is approved for people aged $\geq 65$ years and live-attenuated vaccine by nasal administration is approved for those aged 2-59 years [13]; their use, however, remains infrequent. Of the 47 people aged $\geq 65$ years who were considered immunised in this study, 14 reported that they received adjuvanted vaccine and 19 did not know, while the rest would have received non-adjuvanted vaccine. Overall, 5/141 immunised participants and 5/18 immunised children aged $\leq 10$ years reported intranasal administration. Vaccine effectiveness analysis was not stratified on that basis.

d Immunised people who received the vaccine $<2$ weeks before symptom onset or for whom this was unknown were excluded from the primary vaccine effectiveness analysis. They were included for assessing 'any' immunisation regardless of timing and for comparison with other sources of vaccine coverage. The denominator is therefore shown for 'any' immunisation.

e Children <2 years-old in 2012/13 were excluded from 2011/12 vaccine uptake analysis as they may not have been age-eligible in autumn 2011.

Children 33 years-old in 2012/13 were excluded from 2010/11 vaccine uptake analysis as they may not have been age-eligible in autumn 2010.

g Children $<4$ years-old in 2012/13 were excluded from influenza $\mathrm{A}\left(\mathrm{H}_{1} \mathrm{~N}_{1}\right)$ pdmog vaccine uptake analysis as they may not have been ageeligible in autumn 2009. 
TABLE 3

Laboratory profile of specimens included in primary analysis, interim 2012/13 influenza vaccine effectiveness evaluation, Canada

\begin{tabular}{|c|c|c|c|c|c|c|}
\hline Specimen included & $\begin{array}{c}\text { Alberta } \\
\mathrm{N}=225 \\
\mathrm{n}(\%)\end{array}$ & $\begin{array}{c}\text { British Columbia } \\
\qquad \begin{array}{c}\mathrm{N}=156 \\
\mathrm{n}(\%)\end{array}\end{array}$ & $\begin{array}{c}\text { Manitoba } \\
\mathrm{N}=63 \\
\mathrm{n}(\%)\end{array}$ & $\begin{array}{c}\text { Ontario }^{a} \\
\mathrm{~N}=108 \\
\mathrm{n}(\%)\end{array}$ & $\begin{array}{c}\text { Quebec } \\
N=187 \\
n(\%)\end{array}$ & $\begin{array}{c}\text { Total } \\
\mathrm{N}=739 \\
\mathrm{n}(\%)\end{array}$ \\
\hline Influenza negative & $120(53)$ & $92(59)$ & $46(73)$ & $48(44)$ & $78(42)$ & $384(52)$ \\
\hline Influenza positive & $105(47)$ & $64(41)$ & $17(27)$ & $60(56)$ & $109(58)$ & $355(48)$ \\
\hline A positive & $89(85)$ & $57(89)$ & $14(82)$ & $59(98)$ & $104(95)$ & $323(91)$ \\
\hline B positive & $16(15)$ & $7(11)$ & $3(18)$ & $1(2)$ & $5(5)$ & $32(9)$ \\
\hline \multicolumn{7}{|l|}{ Influenza A positive } \\
\hline $\mathrm{H}_{3} \mathrm{~N}_{2}$ & $81(91)$ & $54(95)$ & $4(29)$ & $54(92)$ & $94(90)$ & $287(89)$ \\
\hline$\left(\mathrm{H}_{1} \mathrm{~N}_{1}\right) \mathrm{pdmog}$ & $4(5)$ & $2(4)$ & $1(7)$ & $4(7)$ & $4(4)$ & $15(5)$ \\
\hline Subtype unknown & $4(5)$ & $1(2)$ & $9(64)$ & $1(2)$ & $6(6)$ & $21(7)$ \\
\hline
\end{tabular}

a Ontario was delayed while awaiting ethics board review, diminishing its contribution to this interim analysis.

TABLE 4

Interim 2012/13 influenza A(H3N2) and overall influenza vaccine effectiveness, Canada

\begin{tabular}{|c|c|c|c|c|}
\hline Analysis scenarios & $\begin{array}{c}A\left(\mathrm{H}_{3} \mathrm{~N}_{2}\right)^{a} \\
\text { VE } \\
(95 \% \mathrm{Cl})\end{array}$ & $\begin{array}{c}\text { Number } \\
\text { Total } \\
\text { (Cases; Vac) } \\
\text { [Controls; Vac] }\end{array}$ & $\begin{array}{c}\text { Any Influenza } \\
\text { VE } \\
(95 \% \mathrm{Cl})\end{array}$ & $\begin{array}{c}\text { Number } \\
\text { Total } \\
\text { (Cases; Vac) } \\
\text { [Controls; Vac] }\end{array}$ \\
\hline \multicolumn{5}{|l|}{ Primary analysis } \\
\hline Crude (unadjusted) ${ }^{\mathrm{b}, \mathrm{c}}$ & $39(10-59)$ & \multirow{11}{*}{$\begin{array}{c}671 \\
(287 ; 45) \\
{[384 ; 90]}\end{array}$} & $45(20-63)$ & \multirow{11}{*}{$\begin{array}{c}739 \\
(355 ; 51) \\
{[384 ; 90]}\end{array}$} \\
\hline \multicolumn{3}{|l|}{ Adjusted for: } & & \\
\hline Age in years $(1-8,9-19,20-49,50-64, \geq 65)$ & $38(4-60)$ & & $46(18-64)$ & \\
\hline Comorbidity (yes/no) ${ }^{\mathrm{b}}$ & $38(7-58)$ & & $43(17-61)$ & \\
\hline Province $(\mathrm{BC}, \mathrm{AB}, \mathrm{MB}, \mathrm{ON}, \mathrm{QC})$ & $46(18-64)$ & & $50(26-66)$ & \\
\hline Specimen collection interval $(\leq 4 \mathrm{~d} / 5-7 \mathrm{~d})$ & $40(10-60)$ & & $46(20-63)$ & \\
\hline Week of specimen collection & $39(9-59)$ & & $45(20-63)$ & \\
\hline Age, comorbidity & $38(3-60)$ & & $45(17-64)$ & \\
\hline Age, comorbidity, province & $45(13-65)$ & & $51(24-68)$ & \\
\hline Age, comorbidity, province, interval & $46(14-66)$ & & $52(26-69)$ & \\
\hline Age, comorbidity, province, interval, week & $45(13-66)$ & & $52(25-69)$ & \\
\hline Sensitivity analysis & & & & \\
\hline Vaccination defined without regard to interval to & set & & & \\
\hline Crude & $38(11-57)$ & & $45(22-62)$ & 767 \\
\hline Fully adjusted $^{d}$ & $38(5-60)$ & $\begin{array}{c}(297 ; 55) \\
{[402 ; 108]}\end{array}$ & $47(21-65)$ & $\begin{array}{l}(365 ; 61) \\
{[402 ; 108]}\end{array}$ \\
\hline Those vaccinated within 2 weeks of symptom on & ed as & & & \\
\hline Unvaccinated; Crude & $38(8-58)$ & 699 & $44(18-61)$ & 767 \\
\hline Unvaccinated; Fully adjusted ${ }^{d}$ & $42(9-63)$ & $\begin{array}{l}(297 ; 45) \\
{[402 ; 90]}\end{array}$ & $48(20-66)$ & $\begin{array}{l}(365 ; 51) \\
{[402 ; 90]}\end{array}$ \\
\hline Vaccinated; Crude & $38(11-57)$ & 699 & $45(22-62)$ & 767 \\
\hline Vaccinated; Fully adjusted ${ }^{d}$ & $38(5-60)$ & $\begin{array}{c}(297 ; 55) \\
{[402 ; 108]}\end{array}$ & $47(21-65)$ & $\begin{array}{c}(365 ; 61) \\
{[402 ; 108]}\end{array}$ \\
\hline Those with unknown comorbidity & & & & \\
\hline Re-coded 'Yes' for comorbidity; Fully adjusted & $45(13-66)$ & $\begin{array}{c}671 \\
(287 ; 45) \\
{[384 ; 90]}\end{array}$ & $51(25-69)$ & $\begin{array}{c}739 \\
(355 ; 51) \\
{[384 ; 90]}\end{array}$ \\
\hline Excluded from analysis & & & & \\
\hline Crude & $38(6-59)$ & 617 & $44(17-62)$ & 683 \\
\hline Fully adjusted $^{d}$ & $44(9-65)$ & $\begin{array}{l}(266 ; 43) \\
{[351 ; 83]}\end{array}$ & $51(23-69)$ & $\begin{array}{l}(332 ; 49) \\
{[351 ; 83]}\end{array}$ \\
\hline Restricted to those with no comorbidity & & & & \\
\hline Crude & $48(14-69)$ & 484 & $53(24-71)$ & 541 \\
\hline Fully adjusted & $60(27-78)$ & $\begin{array}{l}(214 ; 25) \\
{[270 ; 55]}\end{array}$ & $65(39-80)$ & $\begin{array}{l}(271 ; 29) \\
{[270 ; 55]}\end{array}$ \\
\hline
\end{tabular}

AB: Alberta; BC: British Columbia; MB: Manitoba; ON: Ontario; QC: Quebec; Vac: vaccinated - i.e. number of (cases) or [controls] vaccinated; VE: vaccine effectiveness.

a Those with influenza $A$ of unknown subtype were excluded from the $A\left(\mathrm{H}_{3} \mathrm{~N}_{2}\right)$-specific analysis.

b For the primary analysis, those with unknown comorbidity were coded as 'No' but explored in the sensitivity analysis as shown.

c Those immunised $<2$ weeks before symptom onset or from whom a specimen was collected $>7$ days since symptom onset (or for whom these were unknown) were excluded but explored in the sensitivity analysis as shown.

d Adjusted for age, comorbidity, province, interval, week.

e Adjusted for age, province, interval, week. 


\section{FIGURE 3}

Phylogenetic tree of influenza A(H3N2) viruses, Canada, 2012/13 sentinel surveillance system

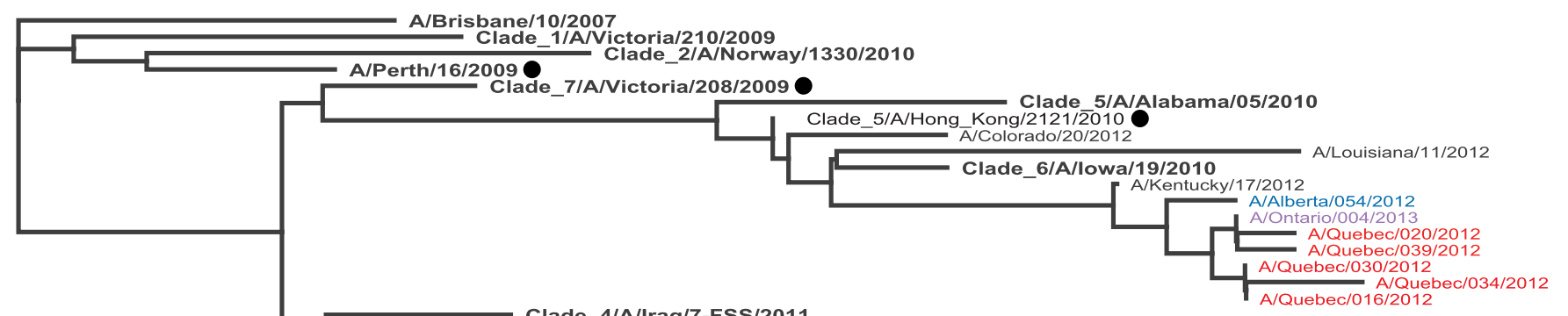

\section{Vaccine and clade strains}

A 2012/13 vaccine reference strain (/Victoria/361/2011)

- Antigenic site comparison strains

British Columbia

Alberta

Manitoba

Ontario

Quebec

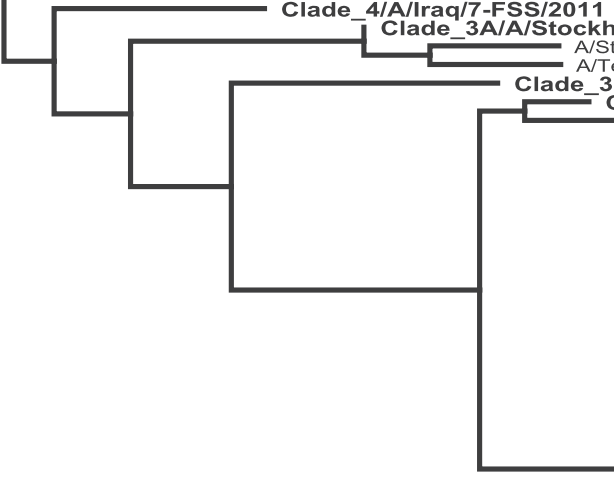
xas/83/2012

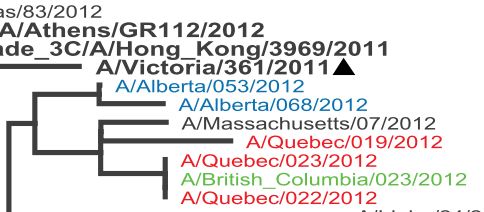

A/ldaho/24/2012

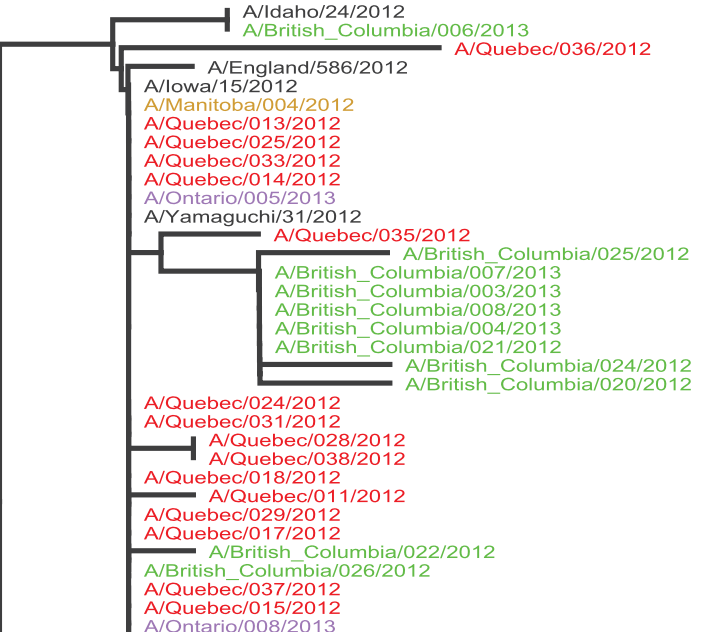

$15 / 2012$

A/Ontario/031/2012

A/Madrid/323/2012

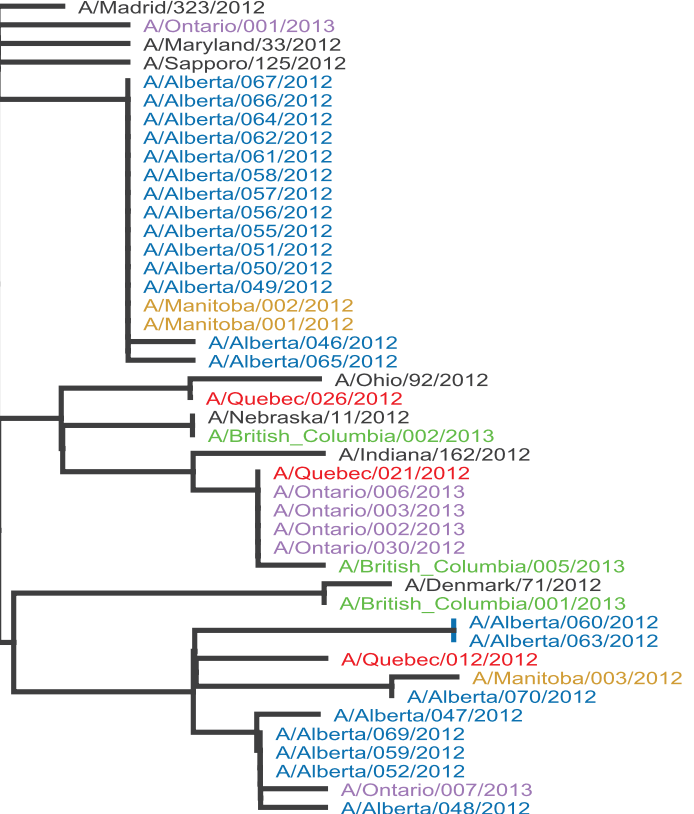

The phylogenetic tree was created by aligning the 82 Canadian sentinel sequences against sequences representative of emerging viral clades as described by the European Centre for Disease Prevention and Control (ECDC) [16] $(n=10)$, $A\left(\mathrm{H}_{3} \mathrm{~N}_{2}\right)$ sequences collected globally between 1 November 2012 and 18 January $2013(n=17)$, and recent vaccine strains $(n=3)$. The global sequences were downloaded from Global Initiative on Sharing Avian Influenza Data (GISAID) by searching for human influenza $A\left(\mathrm{H}_{3} \mathrm{~N}_{2}\right)$ haemagglutinin sequences collected in the specified period (Table 1). 
TABLE 5

Changes in amino acid sequence encoded by haemagglutinin (HA1) gene (antigenic regions) ${ }^{a}$ for subset of 2012/13 Canadian sentinel influenza $\mathrm{A}(\mathrm{H} 3 \mathrm{~N} 2)$ strains relative to reference strains ${ }^{\mathrm{b}}$

\begin{tabular}{|c|c|c|c|c|c|c|c|c|c|c|c|c|c|c|c|c|c|c|c|c|c|c|c|c|c|}
\hline \multirow{2}{*}{\multicolumn{2}{|c|}{$\begin{array}{r}\text { Antigenic site } \\
\text { Amino acid number } \mathrm{HA} 1\end{array}$}} & \multicolumn{4}{|c|}{ C } & \multicolumn{4}{|c|}{ E } & \multirow{2}{*}{\begin{tabular}{|c|}
$D$ \\
121 \\
\end{tabular}} & \multirow{2}{*}{\begin{tabular}{c|}
$\mathrm{A}$ \\
124 \\
\end{tabular}} & \multirow{2}{*}{\begin{tabular}{c|}
$\mathrm{B}$ \\
128 \\
\end{tabular}} & \multicolumn{2}{|c|}{ A } & \multicolumn{5}{|c|}{ B } & \multicolumn{2}{|c|}{ D } & \multicolumn{4}{|c|}{ C } \\
\hline & & 45 & 48 & 53 & 54 & 62 & 67 & 88 & 94 & & & & 142 & 145 & 156 & 157 & 186 & 192 & 198 & 219 & 230 & 278 & 280 & 304 & 312 \\
\hline \multicolumn{2}{|c|}{$\mathrm{A} /$ Perth/16/2009 } & $\mathbf{S}$ & $\mathbf{T}$ & $\mathrm{D}$ & $\mathrm{S}$ & $\mathbf{K}$ & 1 & $\mathrm{~V}$ & $\mathrm{Y}$ & $\mathrm{N}$ & $\mathrm{S}$ & $\mathrm{T}$ & $\mathrm{R}$ & $\mathrm{N}$ & H & $\mathrm{L}$ & G & 1 & A & $S$ & 1 & $\mathrm{~N}$ & $E$ & $A$ & $\mathbf{N}$ \\
\hline \multicolumn{2}{|c|}{ A/Victoria/208/2009 } & $\mathbf{S}$ & $T$ & $\mathrm{D}$ & $\mathrm{S}$ & $\mathrm{E}$ & 1 & $\mathrm{~V}$ & $Y$ & $\mathrm{~N}$ & $\mathrm{~S}$ & $T$ & $R$ & $\mathrm{~N}$ & H & $\mathrm{L}$ & G & 1 & $\mathbf{A}$ & $\mathbf{S}$ & 1 & $\mathrm{~N}$ & $\mathrm{E}$ & A & $\mathbf{N}$ \\
\hline \multicolumn{2}{|c|}{ A/Hong Kong/2121/2010 } & $\mathbf{S}$ & $T$ & $\mathbf{N}$ & $S$ & $\mathrm{E}$ & 1 & V & H & $\mathrm{N}$ & $S$ & $\mathrm{~T}$ & $\mathrm{R}$ & $\mathrm{N}$ & H & $\mathrm{L}$ & G & 1 & $\mathbf{A}$ & $\mathbf{S}$ & $\mathbf{v}$ & $\mathrm{N}$ & $\mathbf{A}$ & A & $\mathbf{N}$ \\
\hline \multicolumn{2}{|c|}{$\mathrm{A} /$ Victoria/361/2011 } & $\mathrm{N}$ & 1 & $\mathrm{D}$ & $\mathrm{s}$ & $\mathrm{E}$ & 1 & $\mathrm{~V}$ & $Y$ & $\mathrm{~N}$ & $\mathrm{~S}$ & $\mathrm{~T}$ & $R$ & $\mathrm{~N}$ & Q & $\mathrm{L}$ & $\mathrm{V}$ & 1 & $\mathrm{~S}$ & $\mathrm{Y}$ & 1 & $\mathrm{~N}$ & $\mathrm{E}$ & A & $\mathrm{S}$ \\
\hline British Columbia & $n$ & & & & & & & & & & & & & & & & & & & & & & & & \\
\hline A/British Columbia/020/2012 ${ }^{\mathrm{d}}$ & 11 & & & & & & & & & & & A & G & $\mathbf{S}$ & H & & G & & & $\mathbf{S}$ & & $\mathbf{K}$ & & & \\
\hline $\mathrm{A} /$ British Columbia/023/2012 ${ }^{\mathrm{d}}$ & 1 & & & & & & & & & & & & & & H & $\mathbf{s}$ & G & & & $\mathbf{s}$ & & K & & & \\
\hline A/British Columbia/002/2013 & 1 & & & & & & & & & & & & & $\mathbf{s}$ & $\mathrm{H}$ & & G & & & $\mathbf{s}$ & & K & & & \\
\hline A/British Columbia/001/2013 & 1 & & & & & & & & & & $\mathbf{N}$ & & & $\mathbf{S}$ & H & & G & & & $\mathbf{S}$ & & K & & & \\
\hline A/British Columbia/005/2013 & 1 & & & & & $\mathbf{K}$ & & & & & & & & $\mathbf{S}$ & H & & G & & & $\mathbf{S}$ & & K & & & \\
\hline Alberta & $\mathrm{n}$ & & & & & & & & & & & & & & & & & & & & & & & & \\
\hline A/Alberta/046/2012 & 14 & & & & & & $\mathbf{v}$ & & & & & & & $\mathbf{S}$ & H & & G & & & $\mathbf{S}$ & & K & & & \\
\hline A/Alberta/047/2012 ${ }^{\mathrm{d}}$ & 6 & & & & & & & & & & & & & $\mathbf{s}$ & H & & G & & & $\mathbf{S}$ & & K & & & \\
\hline A/Alberta/053/2012 ${ }^{\mathrm{d}}$ & 2 & & & & & & & & & & & & & & H & & G & & & $\mathbf{S}$ & & $\mathbf{K}$ & & & \\
\hline A/Alberta/06o/2012 ${ }^{d}$ & 2 & & & & G & & & & & & & & & $\mathbf{S}$ & H & & G & & & $\mathbf{S}$ & & $\mathbf{K}$ & & & \\
\hline A/Alberta/054/2012 ${ }^{\mathrm{e}}$ & 1 & $\mathbf{s}$ & $\mathbf{T}$ & $\mathbf{N}$ & & & & & H & & & & & & H & & G & & A & $\mathbf{S}$ & $\mathbf{v}$ & & A & D & $\mathbf{N}$ \\
\hline Manitoba & $n$ & & & & & & & & & & & & & & & & & & & & & & & & \\
\hline A/Manitoba/001/2012 ${ }^{\mathrm{d}}$ & 2 & & & & & & $\mathbf{v}$ & & & & & & & $\mathbf{s}$ & H & & G & & & $\mathbf{s}$ & & K & & & \\
\hline A/Manitoba/003/2012 ${ }^{\mathrm{d}}$ & 1 & & & & & & & & & & & & & $\mathbf{S}$ & H & & G & & & $\mathbf{S}$ & & K & & & \\
\hline $\mathrm{A} /$ Manitoba/004/2012 ${ }^{\mathrm{d}}$ & 1 & & & & & & & & & & & A & G & $\mathbf{S}$ & H & & G & & & $\mathbf{S}$ & & K & & & \\
\hline Ontario & $\mathrm{n}$ & & & & & & & & & & & & & & & & & & & & & & & & \\
\hline A/Ontario/030/2012 ${ }^{\mathrm{d}}$ & 5 & & & & & & & & & & & & & $\mathbf{s}$ & $\mathrm{H}$ & & G & & & $\mathbf{s}$ & & K & & & \\
\hline A/Ontario/005/2013 & 2 & & & & & & & & & & & A & G & $\mathbf{S}$ & H & & G & & & $\mathbf{S}$ & & K & & & \\
\hline A/Ontario/031/2012 & 2 & & & & & & & & & & $\mathbf{R}$ & A & G & $\mathbf{S}$ & $\mathrm{H}$ & & G & & & $\mathbf{S}$ & & $\mathrm{K}$ & & & \\
\hline A/Ontario/001/2013 & 1 & & & & & & & & & $\mathbf{S}$ & & & & $\mathbf{s}$ & H & & G & & & $\mathbf{s}$ & & $\mathbf{K}$ & & & \\
\hline A/Ontario/004/2013 & 1 & $\mathbf{S}$ & $\mathbf{T}$ & $\mathbf{N}$ & & & & & $\mathbf{H}$ & & & & & & H & & G & & $\mathbf{A}$ & $\mathbf{S}$ & $\mathbf{v}$ & & A & & $\mathbf{N}$ \\
\hline Quebec & $\mathrm{n}$ & & & & & & & & & & & & & & & & & & & & & & & & \\
\hline A/Quebec/011/2012 ${ }^{d}$ & 14 & & & & & & & & & & & A & G & $\mathbf{s}$ & $\mathrm{H}$ & & G & & & $\mathbf{s}$ & & K & & & \\
\hline A/Quebec/028/2012 ${ }^{d}$ & 2 & & & & & & & & & & & A & G & $\mathbf{S}$ & $\mathrm{H}$ & & G & $\mathbf{v}$ & & $\mathbf{s}$ & & $\mathbf{K}$ & & & \\
\hline A/Quebec/019/2012 & 3 & & & & & & & & & & & & & & H & $\mathbf{s}$ & G & & & $\mathbf{S}$ & & $\mathbf{K}$ & & & \\
\hline A/Quebec/021/2012 & 2 & & & & & & & & & & & & & $\mathbf{S}$ & $\mathrm{H}$ & & $\mathbf{G}$ & & & $\mathbf{S}$ & & $K$ & & & \\
\hline A/Quebec/012/2012 ${ }^{d}$ & 1 & & & & & & & $\mathbf{I}$ & & & & & & $\mathbf{S}$ & H & & G & & & $\mathbf{S}$ & & K & & & \\
\hline A/Quebec/020/2012 & 2 & $\mathbf{S}$ & $T$ & $\mathbf{N}$ & & & & & H & & & & & & H & & G & & A & $\mathbf{S}$ & $\mathbf{v}$ & & $\mathbf{A}$ & & $\mathbf{N}$ \\
\hline A/Quebec/016/2012 & 2 & $\mathbf{s}$ & $\mathbf{T}$ & $\mathbf{N}$ & & & & & $\mathrm{H}$ & & & & & & $\mathrm{H}$ & & $\mathbf{G}$ & & $\mathbf{T}$ & $\mathbf{s}$ & $\mathbf{v}$ & & $\mathbf{A}$ & & $\mathbf{N}$ \\
\hline $\mathrm{A} /$ Quebec/034/2012 & 1 & $\mathbf{S}$ & $\mathbf{T}$ & $\mathbf{N}$ & & & & & $\mathbf{Q}$ & & & & & & H & & G & & $\mathbf{T}$ & $\mathbf{S}$ & $\mathbf{v}$ & & A & & $\mathbf{N}$ \\
\hline
\end{tabular}

Bold font signifies amino acid substitution compared with the 2012/13 northern hemisphere vaccine reference strain.

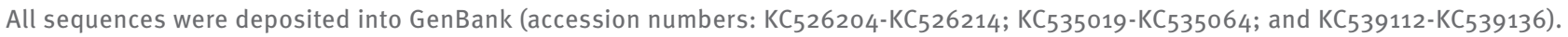

a Antigenic regions A-E comprise 131 amino acid residues [12]. Only the 24 positions in those 131 residues showing mutations in the present study are displayed. British Columbia, Alberta, Manitoba and Ontario sequencing was performed on original specimens; Quebec performed the sequencing on virus isolates.

2012/13 northern hemisphere vaccine reference strain (A/Victoria/361/2011) and other recent vaccine and variant reference strains. 2012/13 northern hemisphere vaccine reference strain.

A total of 75 sentinel sequences clustered within Clade $3 \mathrm{C}$, which also includes the 2012/13 A/Victoria/361/2011 vaccine strain ([16] and Figure 3). Common to each of these 75 sentinel sequences however, were antigenic site mutations compared with the A/Victoria/361/2011 vaccine strain as shown in this table and summarised as follows, with the antigenic site shown in parentheses: Q156H (B), V186G (B), Y219S (D), N278K (C). Of these 75 sequences, 69 also showed N145S (A) while the other four included L157S (B). Of these 69 sequences, 14/22 Alberta and 2/4 Manitoba sequences additionally showed I67V (E) and 11/14 British Columbia, 1/4 Manitoba, 4/10 Ontario and 16/19 Quebec sequences included T128A causing loss of glycosylation site (B) as well as R142G (A) mutations.

e Seven sequences clustered within Clade 6 (A/lowa/19/2010-like; see [16] and Figure 3) with antigenic site mutations compared with the A/ Victoria/361/2011 vaccine strain as shown in this table and additional loss of glycosylation at non-antigenic site N45S (not shown). 
our own adjusted VE estimates did not substantially differ (less than 5-10\%) from our unadjusted VE estimates, assessment of bias and confounding has to be separately undertaken for each dataset. Nevertheless, suboptimal VE for the influenza $A\left(\mathrm{H}_{3} \mathrm{~N}_{2}\right)$ component of the vaccine in both Canada and the US is inconsistent with haemagglutination inhibition characterisation indicating good vaccine match to circulating $\mathrm{A}\left(\mathrm{H}_{3} \mathrm{~N}_{2}\right)$ viruses $[1,2]$. Such discordance between conventional in vitro characterisation of vaccine match by haemagglutination inhibition and epidemiological measures of VE has been noted in previous seasons' estimates from our sentinel network $[6,7,11]$, highlighted also in a recent meta-analysis of other studies, including randomised controlled trials [18].

Molecular markers of virus mutation may offer more insight. It has previously been suggested that a change of at least four AA in two or more HA antigenic sites heralds emergence of virus drift, potentially compromising antibody binding [19]. However, HA antigenicsite maps have been updated and more studies are needed to correlate genetic variation in circulating viruses with epidemiological variation in measured VE $[12,20]$. Not only the number but also the nature and location of AA substitutions are likely to be relevant. Furthermore, hypotheses to explain the variable efficacy of repeat immunisation have included positive and negative interference from pre-existing antibody, with differential effects depending on the antigenic distance across successive vaccine components and circulating strains [21]. We note that a high proportion of participants (91\%) who were immunised this season had also received vaccine the previous season. These virological, host and other factors potentially contributing to suboptimal VE warrant more in-depth evaluation.

Limitations of this surveillance approach to VE estimation have been described previously [6-11]. For our interim analysis, we draw particular attention to small sample size, resulting in wide confidence intervals and variability around the point estimate. Age-specific VE analyses (e.g. children and elderly people) would be of additional important interest - our estimates primarily reflect the prominent contribution of adults 20-49 years of age. However, stratification of VE analysis by age would further reduce the statistical power and precision of estimates in this interim report. The slightly higher VE with restriction to participants without comorbidity (Table 4) may similarly reflect such variability. End-of-season analysis will further expand upon these interim findings and may better support stratified analyses. Although we have assessed vaccine relatedness through gene sequencing of communitybased sentinel viruses available from each province and across the season to date, in this interim assessment the sampling frame for specimen selection was not random or systematic. Bias may result from the preferential inclusion of specimens that demonstrate low cycle threshold values (high RNA levels) or successful virus isolation. These, however, are issues for all laboratory-based influenza surveillance. Finally, in reviewing participant profiles, we identified no obvious signals of bias and in our analysis we adjusted for recognised potential confounders, but ultimately, given the observational design, we cannot rule out other unrecognised influences on the VE estimates.

In summary, our interim findings indicate that the $2012 / 13$ vaccine shows a substantial but suboptimal protection. As such, adjunct protective measures (e.g. antivirals) may be warranted for those at high risk of influenza complications, whether they are vaccinated or not. Interim virus monitoring and VE results may also inform vaccine reformulation for subsequent seasons. Ultimately, however, better understanding of the factors affecting annual influenza VE is needed for improved product development and immunisation programme acceptance in the long term.

\section{Acknowledgements}

The authors gratefully acknowledge the contribution of sentinel sites whose regular submission of specimens and data provide the basis of our analyses. We wish to thank the following for network coordination activities in each province including: Elaine Douglas, Kasim Qureshi (Alberta); Hazel Rona, Tanis Kershaw, Debbie Nowicki, Arielle GoldmanSmith, Alex Henteleff (Manitoba); Romy Olsha, (Ontario); Sophie Auger, Monique Douville Fradet (Quebec). We thank those who provided laboratory support in each province including Virology staff of the British Columbia Public Health Microbiology and Reference, the Alberta Provincial and Public Health Ontario Laboratories and Roy Cole and Kerry Dust of the Cadham Provincial Laboratory (Manitoba) and Joel Ménard and Lyne Désautels of the Laboratoire de santé publique du Québec. We further acknowledge the gene sequencing support provided by Kanti Pabbaraju and Sallene Wong (Alberta), Paul Rosenfeld and Aimin Li (Ontario) and the authors, originating and submitting laboratories of the reference virus sequences from GISAID's EpiFlu Database (www.gisaid.org) (see Table 1).

SMM holds a Canada Research Chair in Pharmacoepidemiology and Vaccine Evaluation, and was supported by an establishment grant from the Manitoba Health Research Council and by Great-West Life, London Life and Canada Life Junior Investigator Award from the Canadian Cancer Society (grant number 2011-700644).

Funding

Funding was provided by the Canadian Institutes of Health Research, the British Columbia Centre for Disease Control, Alberta Health and Wellness, Public Health Ontario, Ministère de la santé et des services sociaux du Québec and the Institut national de santé publique du Québec.

\section{Conflict of interest}

GDS has received research grants from GlaxoSmithKline (GSK) and Sanofi Pasteur and participated in an ad hoc GSK advisory board meeting for an unrelated issue for which travel expenses were reimbursed. SMM has received research grants from GSK, Pfizer and Sanofi Pasteur. JBG has received research grants from GSK and Hoffmann-LaRoche for antiviral resistance studies. MK has received research grants from Roche, Merck, Gen-Probe and Siemens. Salaries 
of TLK and SS provided by a grant of the Canadian Institutes of Health Research. No other authors have competing interests to declare.

\section{Authors' contributions}

Principal investigator (epidemiology): DMS (National and British Columbia); GDS (Quebec); JAD (Alberta); ALW (Ontario); SMM (Manitoba). Principal investigator (laboratory): JBG (Ontario); HC (Quebec); MPP and MK (British Columbia); KF (Alberta); PVC (Manitoba), YL (national). National database coordination: TLK. Data analysis: NZJ and DMS (epidemiology); SS and AE (phylogenetic). Data interpretation: all. Preparation of first draft: DMS. Draft revision and approval: all.

\section{References}

1. Public Health Agency of Canada. FluWatch. [Accessed 26 Jan 2013]. Available from: http://www.phac-aspc.gc.ca/ fluwatch/12-13/index-eng.php

2. Centers for Disease Control and Prevention (CDC). FluView. Atlanta, GA: CDC. [Accessed 26 Jan 2013]. Available from: http://www.cdc.gov/flu/weekly/

3. World Health Organization (WHO). WHO recommendations on the composition of influenza virus vaccines. Geneva: WHO. [Accessed 11 Jan 2013]. Available from: http://www.who.int/ influenza/vaccines/virus/recommendations/en/index.html

4. Janjua NZ, Skowronski DM, De Serres G, Winter A-L, Dickinson JA, Mahmud SM, et al. Component-specific estimates of 201112 influenza vaccine effectiveness based on the Canadian sentinel surveillance system. Tenth Canadian Immunization Conference, 3-5 December 2012; Vancouver, British Columbia, Canada. Poster number P-001.

5. Skowronski DM, Gilbert M, Tweed SA, Petric M, Li Y, Mak A, et al. Effectiveness of vaccine against medical consultation due to laboratory-confirmed influenza: results from a sentinel physician pilot project in British Columbia, 2004-2005. Can Commun Dis Rep. 2005;31:181-91. Available from: http://www. phac-aspc.gc.ca/publicat/ccdr-rmtc/05vol31/dr3118a-eng.php

6. Skowronski DM, Masaro C, Kwindt TL, Mak A, Petric M, Li Y, et al. Estimating vaccine effectiveness against laboratoryconfirmed influenza using a sentinel physician network: results from the $2005-2006$ season of dual $A$ and $B$ vaccine mismatch in Canada. Vaccine. 2007;25(15):2842-51.

7. Skowronski DM, De Serres G, Dickinson J, Petric M, Mak $A$, Fonseca K, et al. Component-specific effectiveness of trivalent influenza vaccine as monitored through a sentinel surveillance network in Canada, 2006-2007. J Infect Dis. 2009;199(2):168-79.

8. Janjua NZ, Skowronski DM, De Serres G, Dickinson J, Crowcroft NS, Taylor M, et al. Estimates of influenza vaccine effectiveness for 2007-08 from Canada's sentinel surveillance system: cross-protection against major and minor variants. J Infect Dis. 2012;205(12):1858-68.

9. Skowronski DM, De Serres G, Crowcroft NS, Janjua NZ, Boulianne N, Hottes TS, et al. Association between the 2008o9 seasonal influenza vaccine and pandemic $\mathrm{H}_{1} \mathrm{~N}_{1}$ illness during spring-summer 2009: four observational studies from Canada. PLoS Med. 2010;7(4):e1000258.

10. Skowronski DM, Janjua NZ, De Serres G, Hottes TS, Dickinson JA, Crowcroft N, et al. Effectiveness of ASo3-adjuvanted pandemic $\mathrm{H}_{1} \mathrm{~N}_{1}$ vaccine: case-control evaluation based on sentinel surveillance system in Canada, autumn 2009. BMJ. 2011;342:C7297. Doi:10.1136/bmj.c7297.

11. Skowronski DM, Janjua NZ, De Serres G, Winter AL, Dickinson JA, Gardy JL, et al. A sentinel platform to evaluate influenza vaccine effectiveness and new variant circulation, Canada 2010-2011 season. Clin Infect Dis. 2012;55(3):332-42.

12. Bush RM, Bender CA, Subbarao K, Cox NJ, Fitch WM. Predicting the evolution of human influenza A. Science. 1999; 286(5446):1921-5.

13. National Advisory Committee on Immunization. Statement on seasonal influenza vaccine for 2012-2013. Can Commun Dis Rep. 2012;38:1-36. Available at: http://www.phac-aspc.gc.ca/ publicat/ccdr-rmtc/12vol38/acs-dcc-2/index-eng.php

14. Statistics Canada. Influenza immunization, less than one year ago by age group and sex (percent). Ottawa: Statistics Canada. [Accessed 30 Jan 2013]. Available from: http://www.statcan. gc.ca/tables-tableaux/sum-som/lo1/csto1/health101b-eng.htm
15. Broemeling AM, Watson DE, Prebtani F. Population patterns of chronic health conditions, co-morbidity and healthcare use in Canada: implications for policy and practice. Healthc Q. 2008;11(3):70-6.

16. European Centre for Disease Prevention and Control (ECDC). Influenza virus characterization. Summary Europe, November 2012. Surveillance report. Stockholm: ECDC. Available from: http://ecdc.europa.eu/en/publications/Publications/influenzavirus-characterisation-CNRL-dec-2012.pdf

17. Centers for Disease Control and Prevention (CDC). Early estimates of seasonal influenza vaccine effectiveness United States, January 2013. MMWR Morb Mortal Wkly Rep. 2013;62:32-5. Available from: http://www.cdc.gov/MMWr/

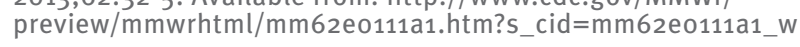

18. Osterholm MT, Kelley NS, Sommer A, Belongia EA. Efficacy and effectiveness of influenza vaccines: a systematic review and metaanalysis. Lancet Infect Dis. 2012; 12(1):36-44.

19. Wilson IA, Cox NJ. Structural basis of immune recognition of influenza virus hemagglutinin. Annu Rev Immunol. 1990;8:737-71.

20. Smith DJ, Lapedes AS, deJong JC, Bestebroer TM, Rimmelzwaan GF, Osterhaus AD, et al. Mapping the antigenic and genetic evolution of influenza virus. Science. 2004;305(5682):371-6.

21. Smith DJ, Forrest S, Ackley DH, Perelson AS. Variable efficacy of repeated annual influenza vaccination. Proc Natl Acad Sci USA. 1999;96(24):14001-6. 